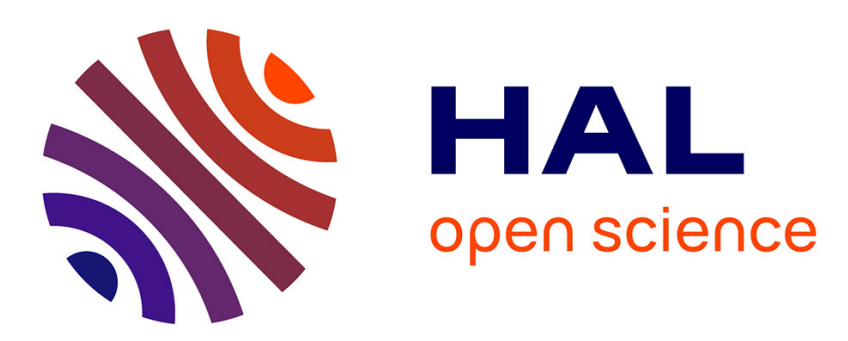

\title{
Reconciling kernel-phase and coronagraphy: new steps towards combining the performance of opposing techniques
}

Romain Laugier, Frantz Martinache, Mamadou N'Diaye, Nick Cvetojevic

\section{- To cite this version:}

Romain Laugier, Frantz Martinache, Mamadou N'Diaye, Nick Cvetojevic. Reconciling kernel-phase and coronagraphy: new steps towards combining the performance of opposing techniques. Optical and Infrared Interferometry and Imaging VII, Dec 2020, Online Only, United States. pp.104, 10.1117/12.2562303 . hal-03396781

\section{HAL Id: hal-03396781 \\ https://hal.science/hal-03396781}

Submitted on 2 Nov 2021

HAL is a multi-disciplinary open access archive for the deposit and dissemination of scientific research documents, whether they are published or not. The documents may come from teaching and research institutions in France or abroad, or from public or private research centers.
L'archive ouverte pluridisciplinaire HAL, est destinée au dépôt et à la diffusion de documents scientifiques de niveau recherche, publiés ou non, émanant des établissements d'enseignement et de recherche français ou étrangers, des laboratoires publics ou privés. 


\title{
Reconciling kernel-phase and coronagraphy: New steps towards combining the performance of opposing techniques
}

\author{
Romain Laugier $^{\mathrm{a}}$, Frantz Martinache ${ }^{\mathrm{b}}$, Mamadou N'Diaye $^{\mathrm{b}}$, and Nick Cvetojevic ${ }^{\mathrm{b}}$

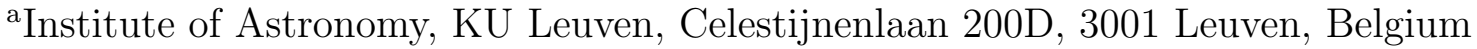 \\ buniversité Côte d'Azur, Observatoire de la Côte d'Azur, CNRS, Laboratoire Lagrange, Bd de \\ l'Observatoire, CS 34229, 06304 Nice cedex 4, France
}

\begin{abstract}
Coronagraphs are powerful tools to probe the direct neighborhood of stars at very high contrasts but their vulnerability to wavefront errors however makes them less efficient for observations at angular separations smaller than two or three resolution elements. In this regime, observables robust to instrumental phase noise, like the closure- and kernel-phase extracted from non-coronagraphic images, have proven capable of effectively picking up moderate contrast features down to the formal diffraction limit. Direct kernel-phase analysis of coronagraphic images is unfortunately not possible in theory. The focal plane mask used in a coronagraph indeed destroys the shift-invariance properties that give meaning to the analysis of their Fourier transform. We nevertheless investigate how techniques initially developed in the context of coronagraphic observations can be applied with kernel-phase to boost the contrast detection limits.

Firstly, inspired by Angular Differential Imaging, we devised a similar method called Angular Differential Kernel to remove static biases from our measurements which are a limiting factor for reaching high contrasts. We present a recent comparative on-sky analysis of its performance using the SCExAO instrument at the Subaru Telescope.

Secondly, we show how pupil plane apodization masks can be used to locally decrease the photon noise in the images, and how their effect translates into the kernel-phase observables, therefore improving the capability of kernel-phase to detect faint companions around nearby stars.
\end{abstract}

Keywords: High contrast, exoplanet, direct imaging, kernel phases

\section{INTRODUCTION}

Coronagraphs have been at the forefront of our efforts to directly detect and characterize exoplanets. The current generation of coronagraphic instruments, represented by SPHERE, ${ }^{1} \mathrm{GPI}^{2}$ and $\mathrm{SCExAO}^{3}$ have now brought a number of scientific results relating to young giant planets around nearby stars. ${ }^{4-6}$ By suppressing the light from the central star and its diffraction pattern, coronagraphs remove the photon noise associated with this pattern across the region of interest. Current coronagraphic designs like vector vortex and $4 \mathrm{QPM}^{7}$ can in theory be made for inner working angles (IWA) down to 1-1.5 $\lambda / D$, where $\lambda$ and $D$ denote the observing wavelength and telescope aperture diameter. These design would allow the discrimination of the planet light from the on-axis light almost perfectly, yet they rely on the perfection of the incoming wavefront. ${ }^{8}$

The practical inner working angle of coronagraphs is therefore limited by what is referred to as speckle noise generated by even the smallest wavefront aberrations at the pupil of the instrument. Techniques intended to suppress the effect of this speckle noise can be traced back to the pupil masking experiments of Fizeau and Stephan ${ }^{9,10}$ and to the event of speckle interferometry in the 1970s by Labeyrie ${ }^{11}$ with the full pupil. The use of closure phases in masked pupil interferometry by Baldwin ${ }^{12}$ or Tuthill ${ }^{13}$ has been generalized to an arbitrary pupil by the works of Martinache ${ }^{14}$ under the name of kernel phases.

Further author information: (Send correspondence to Romain Laugier)

Romain Laugier: E-mail: romain.laugier@kuleuven.be, Telephone: +33681914705 

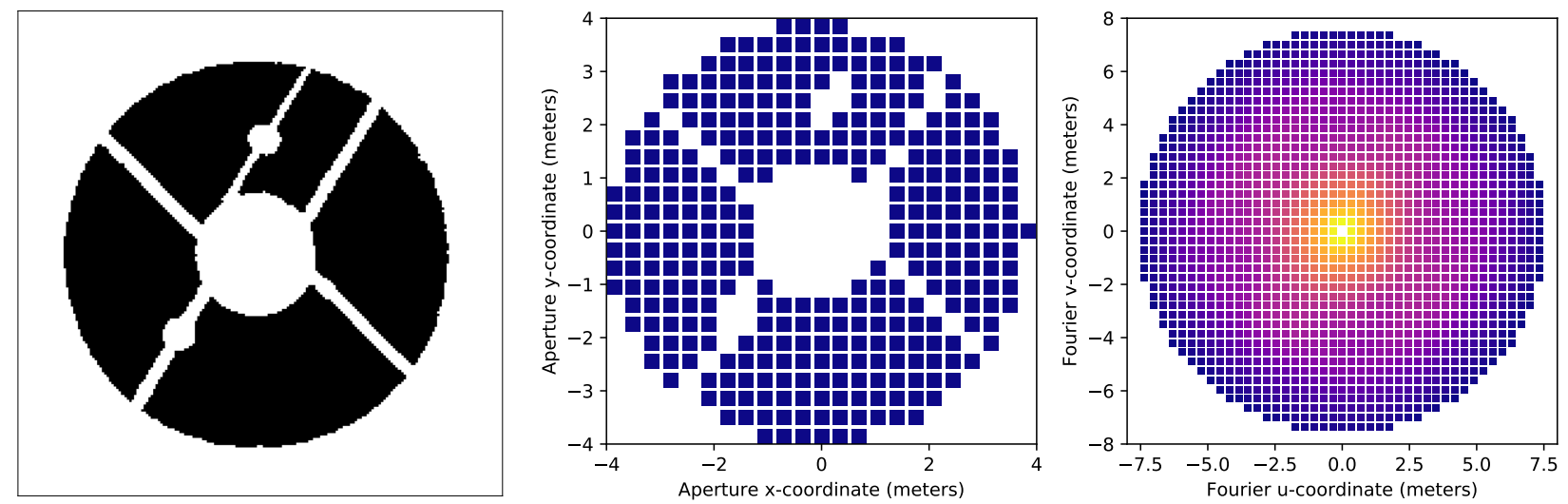

Figure 1. The pupil of the instrument measured as seen in pupil-viewing mode (left), the discrete pupil model built from it (middle) and corresponding UV coverage of the SCExAO pupil as used in section 3 (right). The missing subapertures in the pupil correspond to a mask covering defective actuators of the deformable mirror. Colors illustrate the redundancy of each baseline. ${ }^{15}$

Combination of such speckle noise reduction techniques with coronagraphy would bring significant performance improvements at the smallest separation regime. However, the use of kernel phases in incompatible with the use of coronagraphs. By suppressing the light from on-axis sources, coronagraphs break the convolution relationship. Without this relationship, the Fourier transform of the images loses most of its properties, and cannot be used to produce meaningful kernel phases.

We here show two ways in which both techniques can be brought together to lead to improved contrast performance. The first provides a new way of calibrating closure or kernel phases inspired from angular differential imaging. The second shows how the kernel phases can be used behind shaped pupil coronagraphs, leading to a performance improvement.

\section{KERNEL PHASES}

In the kernel formalism, we use a discrete model of the pupil ${ }^{16}$ to construct both a sampling of the Fourier plane of the image, and the matrix $\mathbf{A}$ that, in the case of small aberrations, models the effect of small pupil plane piston phase errors $\varphi$ on the Fourier phase vector $\boldsymbol{\Phi}$ of the image. Based on this model, a matrix $\mathbf{K}$ can be built that maps the left null space of $\mathbf{A}$, and therefore verifying $\mathbf{K A}=0$. This leads to the suppression of the instrumental phase from the equation to be used in model-fitting:

$$
\mathbf{K} \boldsymbol{\Phi}=\mathbf{K} \boldsymbol{\Phi}^{O}+\mathbf{K A} \varphi+\varepsilon
$$

where $\boldsymbol{\Phi}^{O}$ is the theoretical signal produced by a model object. The term $\varepsilon$ is a residual and constitute the error term.

To simplify the notation, we will use the symbols $\boldsymbol{\kappa}=\mathbf{K} \boldsymbol{\Phi}$ and $\boldsymbol{\kappa}^{O}=\mathbf{K} \boldsymbol{\Phi}^{O}$ to represent respectively the robust observables extracted and the robust observables constructed from the model.

Like for most observations $\varepsilon$ will here be decomposed into two categories:

- fast-evolving errors that will be handled statistically and identified by $\varepsilon_{\text {noise }}$,

- slow-evolving (quasi-static) errors that will be handled by calibration and identified by $\varepsilon_{c a l}$.

\section{ANGULAR DIFFERENTIAL KERNEL PHASES}

\subsection{Calibration}

Calibration relies on evaluating the biases by observing a different target in the same observing conditions. In our case, this bias is additive and can simply be subtracted from the science data to obtain calibrated observation. 
Because of the need for high accuracy, high-contrast imaging requires excellent calibration. This manifests in the need to precisely match observing conditions for the science target and for the calibrator, in terms of time, luminosity, spectral type, on-sky position, etc. .

This makes the calibration a tedious process requiring a significant fraction of the observing time.

In addition, for the case of closure phases and kernel phases, the signature of a companion is antisymmetric, meaning that the detection of a companion around the calibrator may be produced by the presence of a companion on the opposite side of the science target.

\subsection{Angular Differential Imaging (ADI)}

Angular differential imaging ${ }^{17}$ is a very successful calibration technique used in the post-processing of coronagraphic observations. It makes use of the rotation of the field of view at the focus of the instruments observing in fixed pupil mode to distinguish between the biases and the signal of interest.

It uses a long series of images featuring significant field rotation to compute a reference frame through a reduction (median, mean, or other). In this reference the rotating signal of interest is smeared, and the biases appears with high signal-to-noise ratio (SNR). The reference frame is then subtracted from each individual frame to remove the bias. After that, each frame is derotated and summed to improve the SNR on the signal of interest.

\subsection{Pairwise approach}

This approach cannot be used directly since the kernel- or closure-phase signals cannot be de-rotated the way images can. In the special case where the sequence is composed of two frames with extracted observable vectors

$$
\left\{\begin{array}{l}
\kappa_{0}=\kappa_{0}^{O}+\varepsilon_{c a l}+\varepsilon_{n o i s e, 0} \\
\kappa_{1}=\kappa_{1}^{O}+\varepsilon_{\text {cal }}+\varepsilon_{n o i s e, 1}
\end{array},\right.
$$

a valid approach is to build a differential observable:

$$
\boldsymbol{\kappa}_{1}-\boldsymbol{\kappa}_{0}=\boldsymbol{\kappa}_{\delta}^{O}+\underline{\varepsilon}_{\text {eat }}-\varepsilon_{\text {cal }}+\varepsilon_{\delta},
$$

where $\boldsymbol{\kappa}_{\delta}^{O}$ is the differential model observable built from the difference of the corresponding model observables using the known field rotation of each frame, and $\varepsilon_{\delta}$ contains the new error term. Assuming $\varepsilon_{c a l}$ is perfectly correlated between the frames, it cancels perfectly. Assuming the $\varepsilon_{\text {noise }}$ terms are perfectly uncorrelated, the covariance of this error can be built from the sum of the estimated covariances $\operatorname{Cov}\left(\varepsilon_{\text {noise }}\right)$.

\subsection{Generalization}

This same approach can be generalized to a sequence of an arbitrary number of frames by constructing a new observable vector $\boldsymbol{\kappa}_{s}$ the concatenation of the observables extracted from each frame. This allows us to consider the observing sequence as a whole. This prompts to write the effect of a constant bias term:

$$
\boldsymbol{\kappa}_{s}=\kappa_{s}^{O}+\mathbf{U}_{f} \varepsilon_{c a l}+\varepsilon_{s}
$$

The $\boldsymbol{U}_{f}$ that maps an arbitrary constant bias vector $\boldsymbol{\varepsilon}_{\text {cal }}$ corresponds to a stack of blocks that are the Identity matrix.

Following the approach published in Laugier et al., ${ }^{15}$ one can build a projection matrix

$$
\mathbf{L}=\left(\mathbf{I}-\frac{1}{n_{f}} \mathbf{U}_{f} \mathbf{U}_{f}^{T}\right)
$$

where $n_{f}$ is the number of frames in the series. Removing one square block of rows provides the matrix $\mathbf{L}^{\prime}$ of dimension $\left(n_{f}-1\right) \cdot n_{k} \times n_{k}$ that has the additional property of representing a surjective transformation.

Thanks to this property, this new observable $\kappa_{A D K}=\mathbf{L}^{\prime} \boldsymbol{\kappa}_{s}$ can be used with all the statistical processing detailed in Ceau et al. ${ }^{18}$

In the particular case $n_{f}=2$, the $\mathbf{L}^{\prime}$ matrix can be written as two blocks:

$$
\mathbf{L}^{\prime}=\left[\begin{array}{ll}
\frac{1}{2} \mathbf{I} & -\frac{1}{2} \mathbf{I}
\end{array}\right],
$$

which is equivalent to the differential observable proposed in section 3.3. 


\subsection{Experiment}

This process was tested on the SCExAO instrument on the Subaru telescope in Hawaii. SCExAO is an extreme adaptive optics coronagraphic instrument designed for the direct detection of exoplanets. Here, we used the direct imaging mode with the internal near-infrared camera that provides high-frame rate capability during dedicated engineering time to observe the binary star 3 Ser.

The orbit calculated by Horch et al. ${ }^{19}$ place the companion at a separation of $\approx 280$ mas with a contrast in the near infrared of $\Delta H \approx 5$ mag. The observation started at 2019-03-21T13:40:00 and lasted for 40 minutes during its transit, afforded a total field rotation of 35 degrees.

The kernel-phase discrete model, shown in Fig. 1 is composed of 329 sub-apertures of equal transmission that normally generate 781 baselines. However, in order to avoid the problem of $\pi$ radians degeneracy of the phase that appears as a combination of the large NCPA and the phase noise, baselines longer than $7.6 \mathrm{~m}$ were discarded. The remaining 740 baselines produce a total of $n_{k}=412$ kernel phases.

The internal infrared camera (a CRED2 from First Light Imaging) was taking $308 \mu s$ exposures at a frequency of $\approx 3 \mathrm{kHz}$. The frames were coadded two by two to improve the SNR, then a selection was operated based on an empirical Strehl ratio criterion. Because of the severe low wind effect during this sequence, only $5 \%$ of the frames were kept.

Kernel phases were then extracted from the selected frames then binned into field-rotation bins of 1.8 degrees. Only the bins containing more than $3 \times n_{k}$ frames were kept to allow an empirical evaluation of the covariance, resulting in a total of $n_{f}=16$ frames.

For comparison, calibration observations were taken on the star 31 Boo with the same observing parameters and data reduction scheme, and binned into a single angular bin.

\subsection{Results}

We here compare the cases of the raw extracted observables (before calibration), the result of classical calibration, and the observables through the new ADK projection.

The colinearity maps ${ }^{20}$ shown in Fig. 2 provide a human-friendly way to represent the data in the basis of binary companions. In this case, the companion is directly visible in the colinearity maps even before the calibration despite the larger background signal.

The best fit binary model is identified independently for the three methods with the Levenberg-Marquardt algorithm, and the residual is further examined. Colinearity maps of the residual does not suggest the presence of any additional point-source in the scene. In the case of classical calibration, the residual signal is more zonal, which suggests some leftover bias. The minimum $\chi^{2}$ of the model is 1.68 with classical calibration, and 0.98 with ADK, indicating a better fit of the data to this simple binary model.

\section{APODIZED KERNEL PHASES}

\subsection{Arbitrary pupil}

In the small aberration regime provided by the extreme adaptive optics in the infrared, the kernel-phase formalism allows us to create robust observables for an arbitrary pupil. This contrasts with the non-redundant or lessredundant ${ }^{21}$ pupil masks that were often used in less favorable conditions, but required to block $90-95 \%$ of the light.

Since kernel phases can be extracted from observations with arbitrary pupils, they are often used in full-pupil imaging, therefore maximizing the instrument throughput. However, the performance in contrast is directly linked to the photon noise in the image. Here, we investigate the possibility to use an apodizing pupil mask to transform the PSF of the instrument to provide an improved performance with respect to this photon noise limitation.

For the purpose of this experiment, we designed a mask based on the works of Soummer ${ }^{22}$ and Carlotti. ${ }^{23}$ We chose to prioritize solutions with small inner working angles (IWA) where kernel-phases are more interesting, and moderate contrasts. Furthermore, we favored simpler shapes that would be easier to build and translate 

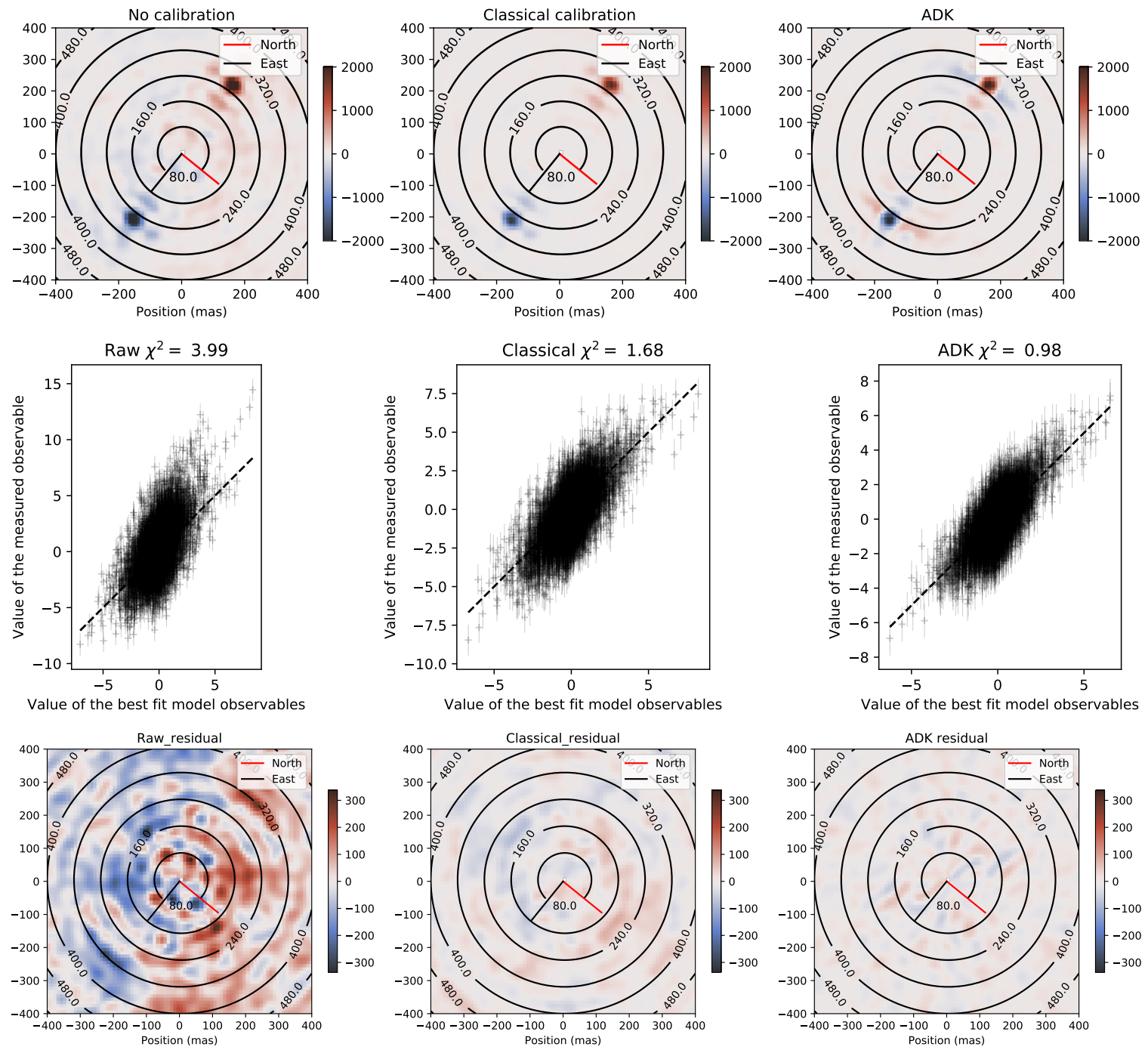

Figure 2. From left to right, in the case of raw observables, classical calibration, and ADK projection. Top row: the colinearity map obtained from the data and showing for the three cases the location of the companion by its positive peak. Middle row: the model correlation diagram of the best fit binary model in the three cases. Bottom row: the colinearity maps obtained on the residual after subtracting the best fit model from the data. This represents how much the residual projects in the basis of binary companions. Classical and ADK approaches provide residual maps with similar that appear similar in amplitude. The $\chi^{2}$ of the best fit model appears significantly better in the case of ADK compared to classical calibration. The orientation of the maps corresponds to the detector at a paralactic angle of $0^{\circ}$. Contours mark the angular separation in mas. ${ }^{15}$ 

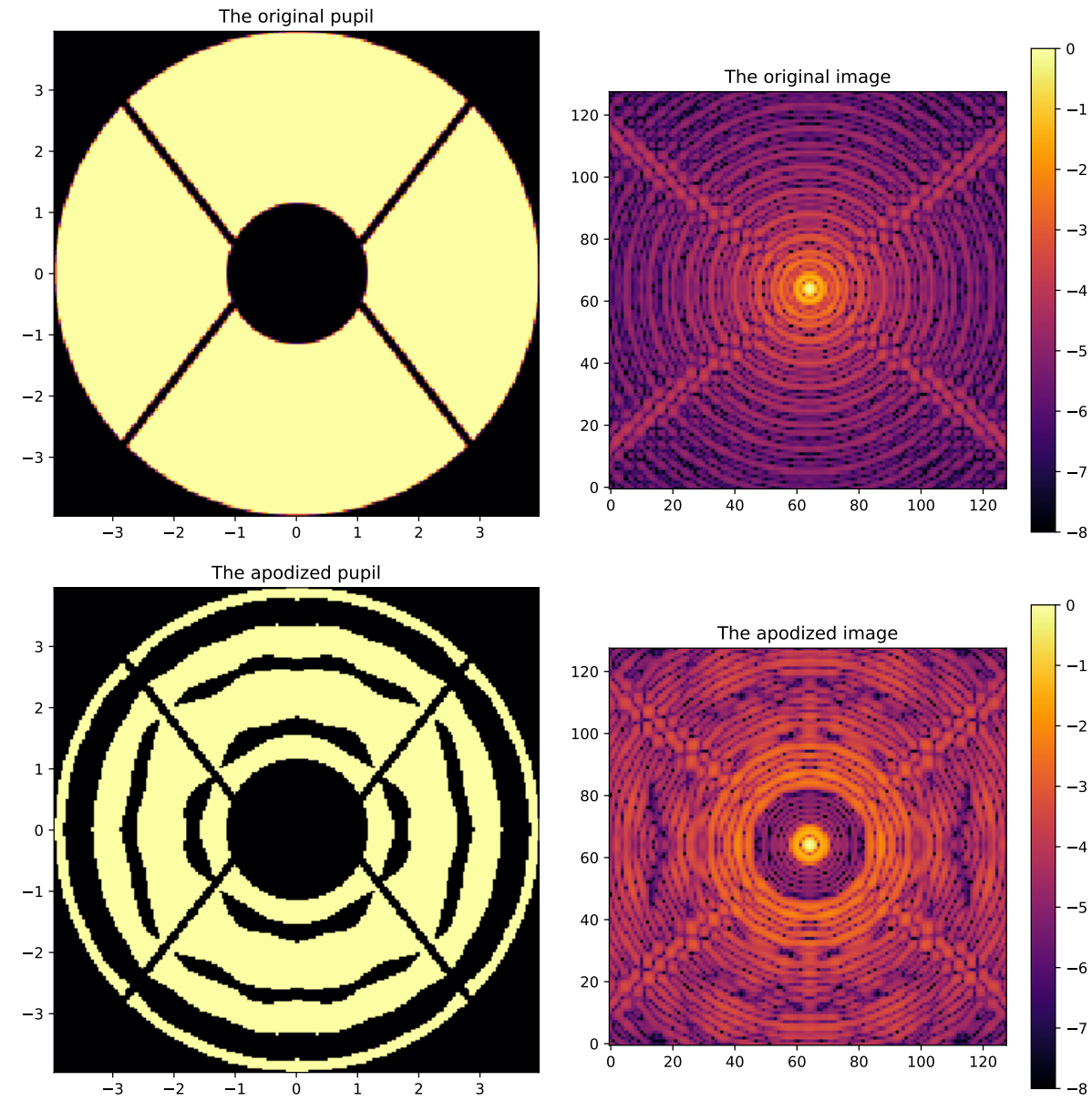

Figure 3. Top: the original SCExAO pupil (left) and PSF in log scale (right). Bottom: the apodizing mask used in this experiment and the corresponding PSF. Note the presence of the dark hole and the brighter wings in the apodized PSF.

into a discrete model for the analysis. We used the pupil of the SCExAO instrument at the Subaru telescope as the constraint for this mask. The proposed solution provides a circular dark hole of $10^{-4}$ contrast from 3 to 6.8 $\lambda / D$, a throughput of 0.64 and is showed in Fig. 3 .

The mask is composed of three modulated rings that modify the power spectrum of the pupil, therefore directly modifying the Optical Transfer Function (OTF) of the instrument. In this new PSF, the outer rings are relatively brighter than in the original PSF.

\subsection{Simulations}

We conducted a comparison of this mask with the original pupil of SCExAO in a controlled simulation environment using the xaosim* simulation package. xaosim uses the Fourier transform of a pupil plane wavefront to simulate aberrated monochromatic images. A companion can then be added by shifting and adding this PSF, before the application of photon noise in the relevant cases. Images are simulated at the wavelength of $1.6 \mu \mathrm{m}$ providing a resolution element of $\lambda / D=40$ mas and with a sampling scale of 16.7 mas/pix.

Since the interest of the simulated dataset lies mostly in the comparison between pupils, we chose to use Kolmogorov phase screens to emulate the residual instrumental pupil-plane phase noise. Completely independent random phase screens are drawn for each image, and their amplitude is adjusted to 50nm RMS in the pupil plane.

${ }^{*}$ https://github.com/fmartinache/xaosim 

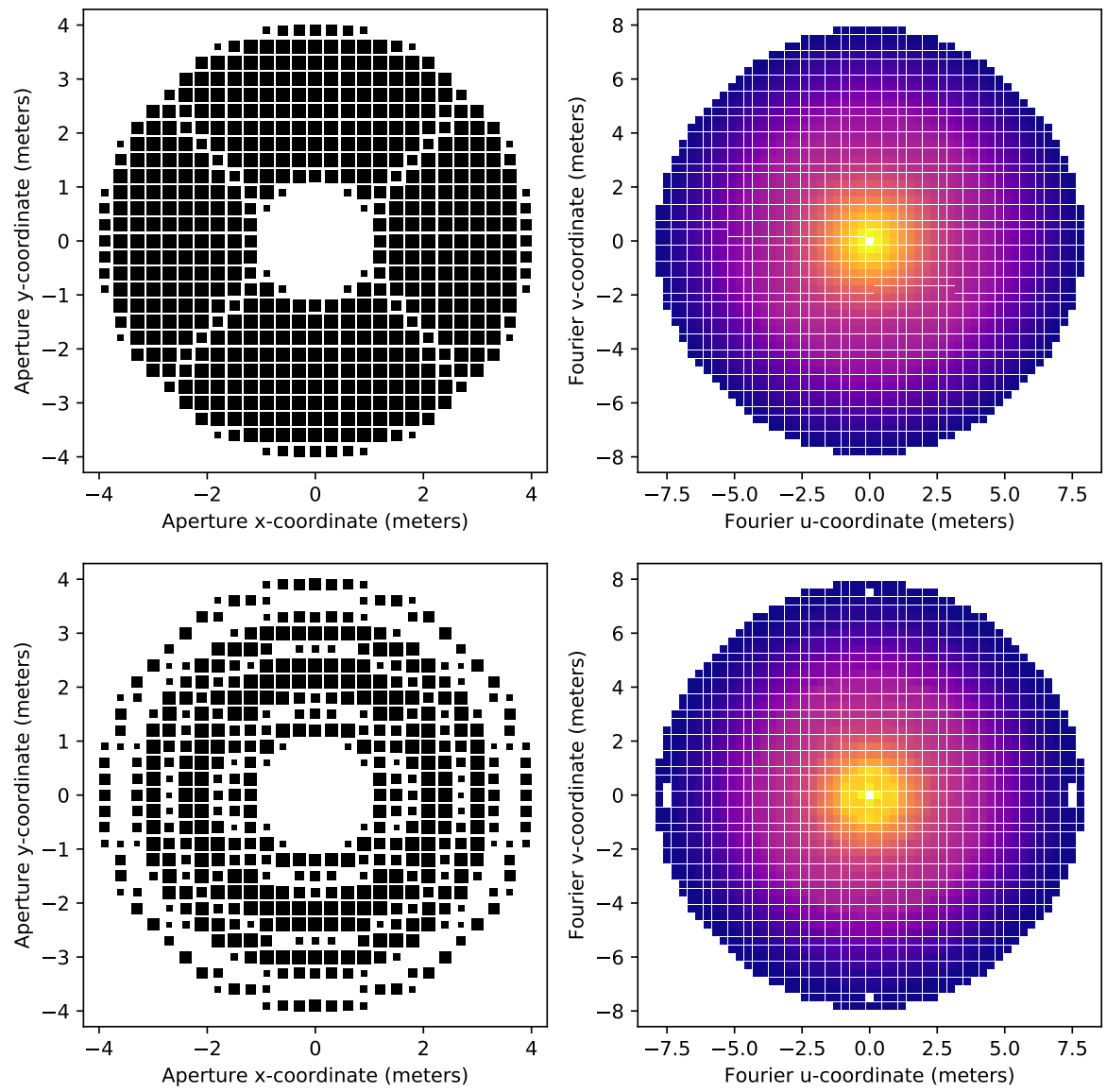

Figure 4. Pupil discrete model (left) and corresponding UV sampling (right) for the original (top) and apodized pupil (bottom). The size of the subaperture markers represent their transmission. The color of the baseline markers represent their redundancy.

Several strategies can be envisioned for the comparison of the pupils: same flux entering the telescope, same flux reaching the sensor, or same peak intensity in the image. Here, we compare images acquired with the same amount of incoming light because it best represents the ultimate limitations of the approach in the context of astrophysics, where we deal with faint targets.

\subsection{Data analysis}

Work presented in Martinache et al. ${ }^{16}$ highlight that the accurate description of the pupil by the model is critical for the performance of the kernel phase analysis, and the complexity of the apodizing pupil represents a challenge for the construction of a pupil model. Here the use of non-binary "grey" models described in this same work and implemented in the package $\mathrm{xara}^{\dagger}$ is helps to provide an adequate representation.

Each subaperture is affected a transmission value corresponding to the integral of the overlap between the subaperture and the free pupil. The baselines longer than the physical diameter of the aperture are discarded as they do not correspond to features that can actually be present in the image data. The description of the models is detailed in Fig. 4 and table 1

For each pupil, 10,000 images are simulated and kernel phase vectors $\boldsymbol{\kappa}$ are extracted with the corresponding discrete model. These vectors are used to estimate the covariance matrix $\boldsymbol{\Sigma}$ of the observables. Following the

\footnotetext{
${ }^{\dagger}$ https://github.com/fmartinache/xara
} 
Table 1. Properties of the pupil and models

\begin{tabular}{c|c|c|c|c} 
Pupil & Transmission & Subapertures & Baselines & Kernel phases \\
\hline Original & 1. & 532 & 1084 & 818 \\
Apodizing & 0.64 & 464 & 1080 & 848
\end{tabular}
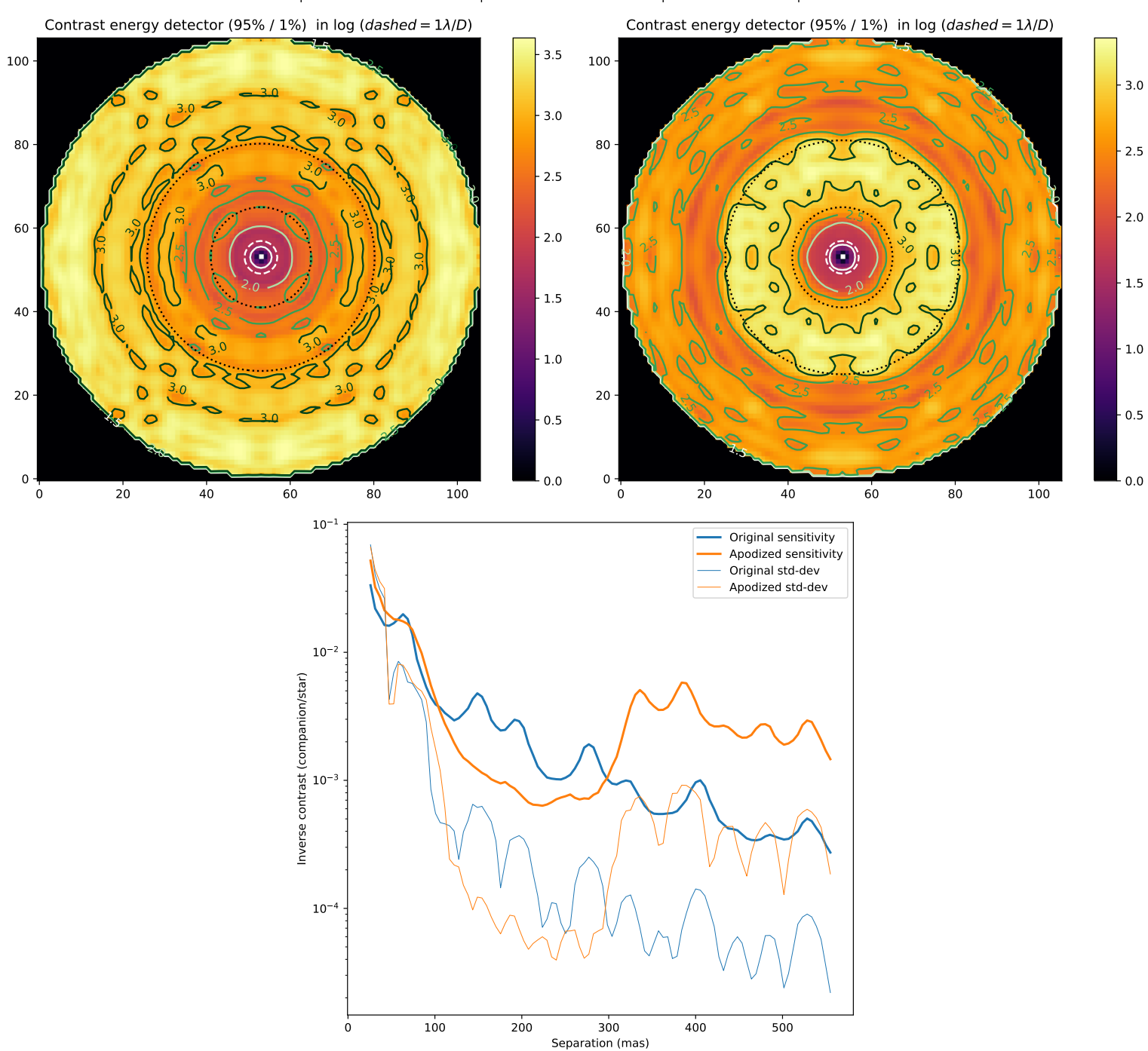

Figure 5. Top: map in relative position around the central star showing in log scale the contrast at which a companion will be detected by a simple energy detector test. Bottom: the mean radial profiles of the same indicators compared to the profile of standard deviation in the images.

approach of Ceau et al., ${ }^{18}$ the covariance matrix is used to produce a whitening matrix $\mathbf{W}=(\boldsymbol{\Sigma})^{-\frac{1}{2}}$. All further analysis is then made with the whitened observables $\mathbf{W} \boldsymbol{\kappa}$.

\subsection{Results}

We use the work of Ceau et al. ${ }^{18}$ to compute the sensitivity of the energy detector test for a false alarm rate of $1 \%$ and detection probability of $95 \%$. The maps of this contrast sensitivity are shown in Fig. 5. They reveal an improvement in sensitivity in most of the dark hole that goes up to a factor 10, leading to a sensitivity to a contrast of $10^{3}$ in most of this region of interest.

These figures correspond to sensitivity for a single image recording a total of $10^{5}$ photons, containing a single realization of the speckle noise, which is therefore not averaged out. This highlights the robustness of kernel 
Table 2. Effect of the apodization on the precision of the astrometric and photometric parameter estimation at a contrast of 530:1 and a separation of 150mas.

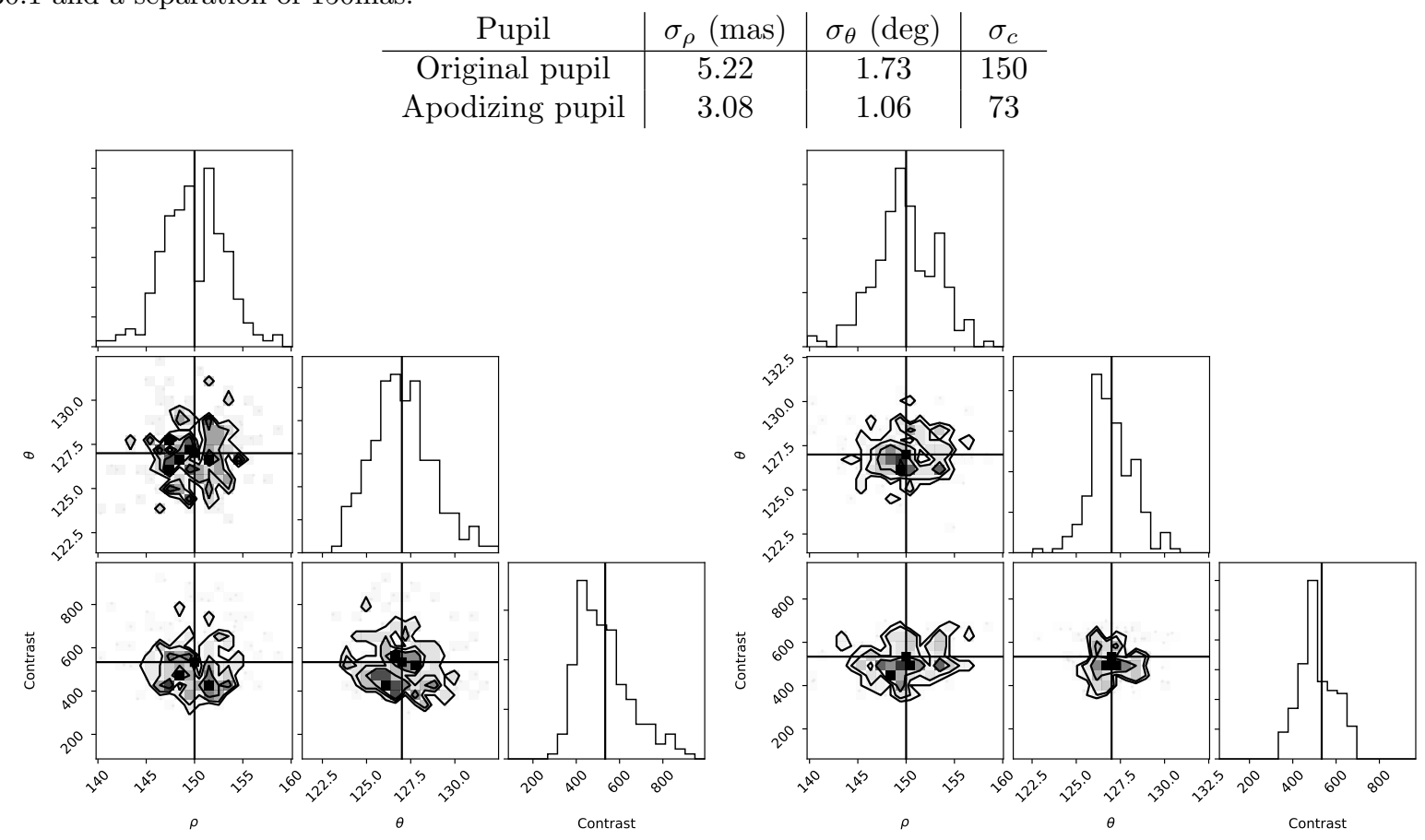

Figure 6. Corner plots showing the distribution of the recovered astrometric parameters. No

phases to quasi-static speckle errors. The use of the mask provides reduction by half of the separation at which this $10^{3}$ contrast can be reached.

The improvements on the precision of the kernel-phase observables also affect the precision of the extraction of the astrometric parameters $\rho$ (angular separation), $\theta$ (position angle) and $c$ (contrast). As an illustration we produced simulated data featuring a binary companion with a contrast of 530:1 and we used Monte Carlo simulations with 500 realizations of independent phase screens and photon noise to evaluate the dispersion of the measurements. For each of the images, the observables were extracted, a colinearity map was used to obtain a rough estimate of the position. Then, a Levenberg-Marquardt algorithm was used to extract the parameters. The standard deviation on 500 realizations in table 2 show a significant improvement of precision of all the parameters.

The mask showed here has been laser-cut and installed in the pupil wheel of SCExAO (Fig. 7). The evaluation of the performance of this approach on sky has not yet been completed, but the simulations show promising results.

\section{CONCLUSIONS}

Coronagraphy and kernel phases cannot be combined directly, but some of the techniques that were developed for coronagraphy are still relevant to kernel-phase observations and can improve their performance. The approach to calibration we called angular differential kernel is a simple projection that can be used both in the case of masked aperture and in the case of full pupil kernel-phase observations and that can alleviate the need for calibration observations. Combined with improvements in the discrete model brought with Martinache et al. 2020, ${ }^{16}$ the kernel-phase approach is being pushed closer to its theoretical limit: the photon noise.

The fact that kernel phases can be extracted from data taken with an arbitrary pupil gives the opportunity to work with pupils optimized for particular applications. In the case described here, the pupil transmission is optimized to maximize the throughput yet provide a circular dark hole in the PSF. Thanks to the use of whitening transform, this property translates into an improvement of performance of high contrast detection 

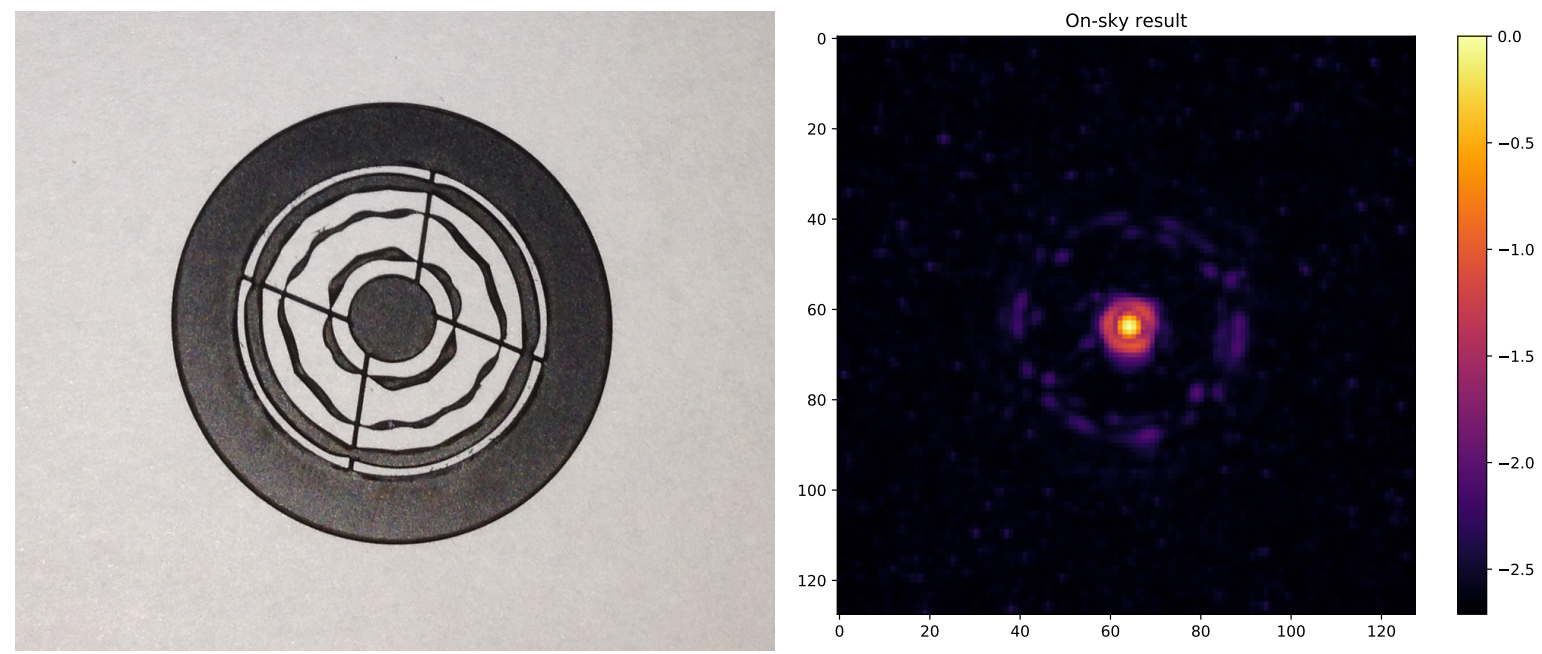

Figure 7. Left: the mask laser-cut for the instrument SCExAO. Right: log-scale image of Vega obtained by lucky imaging in poor seeing conditions during commissioning.

tests. This shows that this theoretical photon noise limit may still be pushed further by simple modifications of the pupil. Pending on-sky validation of this approach should confirm how much it pushes both our capability to detect lower-mass companions at small separations, and improve the precision of astrometric and photometric measurements of known companions in this separation range.

\section{ACKNOWLEDGMENTS}

KERNEL has received funding from the European Research Council (ERC) under the European Union's Horizon 2020 research and innovation program (grant agreement CoG - 683029). Based in part on data collected at Subaru Telescope, which is operated by the National Astronomical Observatory of Japan. The development of SCExAO was supported by the Japan Society for the Promotion of Science (Grant-in-Aid for Research \#23340051, \#26220704, \#23103002, \#19H00703 \& \#19H00695), the Astrobiology Center of the National Institutes of Natural Sciences, Japan, the Mt Cuba Foundation and the director's contingency fund at Subaru Telescope. The authors wish to recognise and acknowledge the very significant cultural role and reverence that the summit of Maunakea has always had within the indigenous Hawaiian community. We are most fortunate to have the opportunity to conduct observations from this mountain.

\section{REFERENCES}

[1] Beuzit, J.-L., Feldt, M., Dohlen, K., Mouillet, D., Puget, P., Wildi, F., Abe, L., Antichi, J., Baruffolo, A., Baudoz, P., Boccaletti, A., Carbillet, M., Charton, J., Claudi, R., Downing, M., Fabron, C., Feautrier, P., Fedrigo, E., Fusco, T., Gach, J.-L., Gratton, R., Henning, T., Hubin, N., Joos, F., Kasper, M., Langlois, M., Lenzen, R., Moutou, C., Pavlov, A., Petit, C., Pragt, J., Rabou, P., Rigal, F., Roelfsema, R., Rousset, G., Saisse, M., Schmid, H.-M., Stadler, E., Thalmann, C., Turatto, M., Udry, S., Vakili, F., and Waters, R., "SPHERE: a 'Planet Finder' instrument for the VLT," Proc of SPIE 7014, 701418 (2008).

[2] Macintosh, B., Graham, J. R., Ingraham, P., Konopacky, Q., Marois, C., Perrin, M., Poyneer, L., Bauman, B., Barman, T., Burrows, A. S., Cardwell, A., Chilcote, J., De Rosa, R. J., Dillon, D., Doyon, R., Dunn, J., Erikson, D., Fitzgerald, M. P., Gavel, D., Goodsell, S., Hartung, M., Hibon, P., Kalas, P., Larkin, J., Maire, J., Marchis, F., Marley, M. S., McBride, J., Millar-Blanchaer, M., Morzinski, K., Norton, A., Oppenheimer, B. R., Palmer, D., Patience, J., Pueyo, L., Rantakyro, F., Sadakuni, N., Saddlemyer, L., Savransky, D., Serio, A., Soummer, R., Sivaramakrishnan, A., Song, I., Thomas, S., Wallace, J. K., Wiktorowicz, S., and Wolff, S., "First light of the Gemini Planet Imager," Proceedings of the National Academy of Sciences of the United States of America 111(35), 12661-12666 (2014). 
[3] Jovanovic, N., Martinache, F., Guyon, O., Clergeon, C., Singh, G., Kudo, T., Garrel, V., Newman, K., Doughty, D., Lozi, J., Males, J., Minowa, Y., Hayano, Y., Takato, N., Morino, J., Kuhn, J., Serabyn, E., Norris, B., Tuthill, P., Schworer, G., Stewart, P., Close, L., Huby, E., Perrin, G., Lacour, S., Gauchet, L., Vievard, S., Murakami, N., Oshiyama, F., Baba, N., Matsuo, T., Nishikawa, J., Tamura, M., Lai, O., Marchis, F., Duchene, G., Kotani, T., and Woillez, J., "The Subaru Coronagraphic Extreme Adaptive Optics System: Enabling High-Contrast Imaging on Solar-System Scales," Publications of the Astronomical Society of the Pacific 127(955), 890-910 (2015).

[4] Macintosh, B., Graham, J. R., Barman, T., De Rosa, R. J., Konopacky, Q., Marley, M. S., Marois, C., Nielsen, E. L., Pueyo, L., Rajan, A., Rameau, J., Saumon, D., Wang, J. J., Patience, J., Ammons, M., Arriaga, P., Artigau, E., Beckwith, S., Brewster, J., Bruzzone, S., Bulger, J., Burningham, B., Burrows, A. S., Chen, C., Chiang, E., Chilcote, J. K., Dawson, R. I., Dong, R., Doyon, R., Draper, Z. H., Duchêne, G., Esposito, T. M., Fabrycky, D., Fitzgerald, M. P., Follette, K. B., Fortney, J. J., Gerard, B., Goodsell, S., Greenbaum, A. Z., Hibon, P., Hinkley, S., Cotton, T. H., Hung, L. W., Ingraham, P., Johnson-Groh, M., Kalas, P., Lafreniere, D., Larkin, J. E., Lee, J., Line, M., Long, D., Maire, J., Marchis, F., Matthews, B. C., Max, C. E., Metchev, S., Millar-Blanchaer, M. A., Mittal, T., Morley, C. V., Morzinski, K. M., Murray-Clay, R., Oppenheimer, R., Palmer, D. W., Patel, R., Perrin, M. D., Poyneer, L. A., Rafikov, R. R., Rantakyrö, F. T., Rice, E. L., Rojo, P., Rudy, A. R., Ruffio, J. B., Ruiz, M. T., Sadakuni, N., Saddlemyer, L., Salama, M., Savransky, D., Schneider, A. C., Sivaramakrishnan, A., Song, I., Soummer, R., Thomas, S., Vasisht, G., Wallace, J. K., Ward-Duong, K., Wiktorowicz, S. J., Wolff, S. G., and Zuckerman, B., "Discovery and spectroscopy of the young jovian planet 51 Eri b with the Gemini Planet Imager," Science 350(6256), 64-67 (2015).

[5] Chauvin, G., Desidera, S., Lagrange, A. M., Vigan, A., Gratton, R., Langlois, M., Bonnefoy, M., Beuzit, J. L., Feldt, M., Mouillet, D., Meyer, M., Cheetham, A., Biller, B., Boccaletti, A., D'Orazi, V., Galicher, R., Hagelberg, J., Maire, A. L., Mesa, D., Olofsson, J., Samland, M., Schmidt, T. O., Sissa, E., Bonavita, M., Charnay, B., Cudel, M., Daemgen, S., Delorme, P., Janin-Potiron, P., Janson, M., Keppler, M., Le Coroller, H., Ligi, R., Marleau, G. D., Messina, S., Mollière, P., Mordasini, C., Müller, A., Peretti, S., Perrot, C., Rodet, L., Rouan, D., Zurlo, A., Dominik, C., Henning, T., Menard, F., Schmid, H. M., Turatto, M., Udry, S., Vakili, F., Abe, L., Antichi, J., Baruffolo, A., Baudoz, P., Baudrand, J., Blanchard, P., Bazzon, A., Buey, T., Carbillet, M., Carle, M., Charton, J., Cascone, E., Claudi, R., Costille, A., Deboulbe, A., De Caprio, V., Dohlen, K., Fantinel, D., Feautrier, P., Fusco, T., Gigan, P., Giro, E., Gisler, D., Gluck, L., Hubin, N., Hugot, E., Jaquet, M., Kasper, M., Madec, F., Magnard, Y., Martinez, P., Maurel, D., Le Mignant, D., Möller-Nilsson, O., Llored, M., Moulin, T., Origné, A., Pavlov, A., Perret, D., Petit, C., Pragt, J., Puget, P., Rabou, P., Ramos, J., Rigal, R., Rochat, S., Roelfsema, R., Rousset, G., Roux, A., Salasnich, B., Sauvage, J. F., Sevin, A., Soenke, C., Stadler, E., Suarez, M., Weber, L., Wildi, F., Antoniucci, S., Augereau, J. C., Baudino, J. L., Brandner, W., Engler, N., Girard, J., Gry, C., Kral, Q., Kopytova, T., Lagadec, E., Milli, J., Moutou, C., Schlieder, J., Szulágyi, J., Thalmann, C., and Wahhaj, Z., "Discovery of a warm, dusty giant planet around HIP 65426," Astronomy and Astrophysics 605, 1-9 (2017).

[6] Mesa, D., Keppler, M., Cantalloube, F., Rodet, L., Charnay, B., Gratton, R., Langlois, M., Boccaletti, A., Bonnefoy, M., Vigan, A., Flasseur, O., Bae, J., Benisty, M., Chauvin, G., De Boer, J., Desidera, S., Henning, T., Lagrange, A. M., Meyer, M., Milli, J., Müller, A., Pairet, B., Zurlo, A., Antoniucci, S., Baudino, J. L., Brown Sevilla, S., Cascone, E., Cheetham, A., Claudi, R. U., Delorme, P., D’Orazi, V., Feldt, M., Hagelberg, J., Janson, M., Kral, Q., Lagadec, E., Lazzoni, C., Ligi, R., Maire, A. L., Martinez, P., Menard, F., Meunier, N., Perrot, C., Petrus, S., Pinte, C., Rickman, E. L., Rochat, S., Rouan, D., Samland, M., Sauvage, J. F., Schmidt, T., Udry, S., Weber, L., and Wildi, F., "VLT/SPHERE exploration of the young multiplanetary system PDS70," Astronomy and Astrophysics 632, 1-12 (2019).

[7] Guyon, O., Pluzhnik, E. A., Kuchner, M. J., Collins, B., and Ridgway, S. T., "Theoretical Limits on Extrasolar Terrestrial Planet Detection with Coronagraphs," The Astrophysical Journal Supplement Series 167(1), 81-99 (2006).

[8] Guyon, O., "Limits of Adaptive Optics for high contrast imaging," (2005).

[9] Fizeau, H., "Note relative au prix Bordin, Rapport du concours de l'année 1867," Comptes rendus de l'Académie des Sciences 66, 932-4 (1864). 
[10] Stephan, E., "Comptes rendus hebdomadaires des séances de l'Académie des sciences / publiés ... par MM . les secrétaires," Comptes Rendus Hebdomadères de l'Académie des Sciences LXXVII(1), 1008-1012 (1874).

[11] Labeyrie, A., "Attainment of Diffraction Limited Resolution in Large Telescopes by Fourier Analysing Speckle Patterns in Star Images," Astronomy 83 Astrophysics 6, 85 (1970).

[12] Baldwin, J. E., Haniff, C. A., Mackay, C. D., and Warner, P. J., "Closure phase in high-resolution optical imaging," Nature 320(6063), 595-597 (1986).

[13] Tuthill, P. G., Monnier, J. D., Danchi, W. C., and Haniff, C. A., "Michelson interferometry with Keck I," Astronomical Interferometry 3350, 839 (1998).

[14] Martinache, F., "Kernel Phase in Fizeau Interferometry," The Astrophysical Journal 724, 464 (2010).

[15] Laugier, R., Martinache, F., Cvetojevic, N., Mary, D., Ceau, A., Diaye, M. N., Lozi, J., Guyon, O., and Lopez, C., "Angular differential kernel phases," Astronomy \& Astrophysics 636(April 2020) (2020).

[16] Martinache, F., Ceau, A., Laugier, R., Kammerer, J., N'DIaye, M., Mary, D., Cvetojevic, N., and Lopez, C., "Kernel-phase analysis: Aperture modeling prescriptions that minimize calibration errors," Astronomy and Astrophysics 636, 1-12 (2020).

[17] Marois, C., Lafreniere, D., Doyon, R., Macintosh, B., and Nadeau, D., "Angular Differential Imaging: A Powerful High-Contrast Imaging Technique," The Astrophysical Journal 641(1), 556-564 (2006).

[18] Ceau, A., Mary, D., Greenbaum, A., Martinache, F., Sivaramakrishnan, A., Laugier, R., and N'Diaye, M., "Kernel-phase detection limits: Hypothesis testing and the example of JWST NIRISS full-pupil images," Astronomy and Astrophysics 630, 1-10 (2019).

[19] Horch, E. P., Van Belle, G. T., Davidson, J. W., Ciastko, L. A., Everett, M. E., and Bjorkman, K. S., "Observations of binary stars with the differential speckle survey instrument. VI. Measures during 2014 at the discovery channel telescope," Astronomical Journal 150(5), 151 (2015).

[20] Laugier, R., Martinache, F., Ceau, A., Mary, D., N'Diaye, M., and Beuzit, J. L., "Recovering saturated images for high dynamic kernel-phase analysis: Application to the determination of dynamical masses for the system Gl 494AB," Astronomy and Astrophysics 623, 1-8 (2019).

[21] Tuthill, P. G., Lacour, S., Amico, P., Ireland, M. J., Norris, B., Stewart, P., Evans, T. M., Kraus, A. L., Lidman, C., Pompei, E., and Kornweibel, N., "Sparse aperture masking (SAM) at NAOS/CONICA on the VLT," Ground-based and Airborne Instrumentation for Astronomy III. Edited by McLean 7735, 77351O77351O-11 (2010).

[22] Soummer, R., Pueyo, L., Sivaramakrishnan, A., and Vanderbei, R. J., "Fast computation of Lyot-style coronagraph propagation," 6269 (2006).

[23] Carlotti, A., Vanderbei, R., and Kasdin, N. J., "Optimal pupil apodizations of arbitrary apertures for high-contrast imaging," Optics Express 19(27), 26796 (2011). 